Revista Iberoamericana de

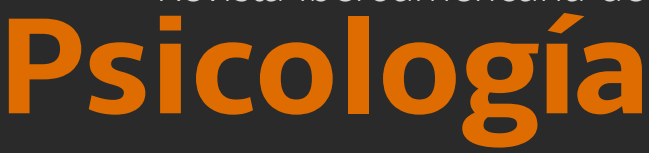

ISSN-I: 2027-1786 | e-ISSN: 2500-6517 Publicación Cuatrimestral

ID: 2027-1786.rip.11105

\section{Title: Professional and private drivers from Bogotá}

Subtitle: The diagnose of sociodemographic conditions and general well-being

Título: Conductores profesionales y conductores particulares bogotanos

Subtítulo: Diagnóstico de condiciones sociodemográficas y bienestar general [análisis exploratorio]

Alt Title / Título alternativo:

[es]

Análisis exploratorio y comparativo de conductores profesionales y conductores particulares bogotanos: diagnóstico de condiciones sociodemográficas y bienestar general

Author (s) / Autor (es):

Serge Rodríguez, Ruiz Pérez, \& Gómez Díaz Keywords / Palabras Clave:

road safety; epidemiology; professional drivers; health conductores profesionales; salud

Submited: 2018-03-05

Acepted: 2018-04-17

\section{Resumen}

A pesar que los accidentes de tráfico son reconocidos mundialmente como un problema de salud pública y que en Colombia representan la segunda causa de muerte violenta, hay una falta de investigación en torno al tema, hecho que señala la importancia de iniciar acciones concretas. En consonancia con lo anterior, el objetivo de la presente investigación fue estudiar dos tipos de población de conductores, a saber,

conductores profesionales y particulares en la ciudad de Bogotá, mediante un análisis y comparación de su situación sociodemográfica y de salud general. Con tales fines, una investigación de corte transversal fue llevada a cabo, en la que participaron

500 conductores (250 profesionales y 250 particulares) colombianos entre los 16 y los

72 años de edad. Los resultados revelan que los conductores profesionales tienen más edad, más experiencia de conducción, ingresos salariales inferiores, menores niveles educativos y reportan un mayor número de accidentes en comparación con los conductores particulares. Por su parte,

los conductores particulares presentan

peores índices de salud, bienestary menos habilidades en la conducción. Estos hallazgos indican tendencias sociodemográficas ya reportadas en la literatura para los conductores profesionales, pero divergen en

cuanto a salud y bienestar. En conclusión, se requieren más estudios para aumentar el conocimiento sobre diferencias entre tipos de conductores y sobre el papel de los factores

de riesgo y de protección con relación a la accidentalidad vial, con miras a su prevención y reducción.

\section{Abstract}

Even though traffic collisions (motor vehicle collisions (MVC) or traffic accidents) are recognized worldwide as a public health problem and are in Colombia the second cause of violent deaths, there is a lack of research on the topic, which is not only a matter of concern but also points out the importance of initiating concrete actions. Consequently, the aim of the present research is to study two types of populations of drivers,

that is, professional and particular drivers in

Bogotá, through an analysis and comparison of their sociodemographic situation and their general health conditions. In order to do that a cross-sectional investigation was carried out, in which 500 Colombian drivers (250 professional drivers and 250 particular drivers), with ages between 16 and 72 years, participated. The results showed that professional drivers are older, have more driving experience, lower income, and lower educational levels, and report a higher number of accidents/collisions than particular drivers. As for particular drivers, they have worse rates of health, welfare, and less driving skills.

These findings indicate sociodemographic trends already reported in the literature on professional drivers but diverge regarding health and well-being. In conclusion, further studies are required in order to enhance knowledge about the differences between typologies of drivers and about the role of the risk and protective factors regarding road accidents, in order to improve prevention and to reduce the number of collisions.
Citar como:

Serge Rodríguez, A. C., Ruiz Pérez, J. I., \& Gómez Díaz, I. A. (2018). Conductores profesionales y conductores particulares bogotanos: Diagnóstico de condiciones sociodemográficas y bienestar general [análisis exploratorio]. Revista Iberoamericana de Psicología issn-l:2027-1786, 11 (1), 45-54. Obtenido de: https://revistas.iberoamericana.edu.co/index.php/ripsicologia/article/view/1336
Andrea Cecilia Serge Rodríguez, PhD Msc MA Psi ORCID: $\quad$ 0000-0001-8369-8499

Source | Filiacion:

Universidad de Valencia

BIO:

PhD (c). Investigación en Psicología

Ms. Psicología, y Ps.

Ms. Investigación Social Interdisciplinaria

Ms. Gestión, Toma de Decisiones y Resolución

de Conflictos

City I Ciudad:

Valencia [es]

e-mail:

andrea.serge@uv.es
José Ignacio Ruiz Pérez, PhD MSc

ORCID: $\quad$ 0000-0002-8996-9736

Source I Filiacion

Universidad Nacional de Colombia

BIO:

PhD Psicología Ms. Criminología

City I Ciudad:

Bogotá DC [co]

e-mail:

jose ignacioru@hotmail.com
Iván Andrés Gómez Díaz, Psi

Source I Filiacion:

Universidad El Bosque

BIO:

Psicólogo

City I Ciudad:

Bogotá DC [Co]

e-mail:

igomezd@unbosque.edu.co 


\title{
Conductores profesionales y conductores particulares bogotanos Diagnóstico de condiciones sociodemográficas y bienestar general [análisis exploratorio]
}

Professional and private drivers from Bogotá: the diagnose of sociodemographic conditions and general well-being

\author{
Andrea Cecilia Serge Rodríguez \\ José Ignacio Ruiz Pérez \\ Iván Andrés Gómez Díaz
}

\section{Introducción}

Los accidentes de tráfico han sido identificados como problema grave en el ámbito de la salud pública debido a sus consecuencias mortales y al gran número de víctimas y lesiones asociadas (Gopalakrishnan, 2012). Se estima que alrededor de 1.2 millones de personas perecen a causa de esta problemática, y que los heridos pueden llegar a 50 millones en todo el mundo (Mcllvenny, 2006). Debido a lo anterior, este fenómeno sigue siendo preocupante para los gobiernos, a pesar de los diversos esfuerzos realizados en este campo (Tolon, Lastra, \& Flores, 2013).

El caso colombiano no difiere mucho del panorama global, pues es posible afirmar que es muy preocupante. Durante el periodo comprendido entre 2002-2012 se registraron aproximadamente $\mathbf{6 2 , 0 0 0}$ fallecidos y alrededor de $\mathbf{4 4 3 , 0 0 0}$ lesionados, y entre los años 2009 y 2011 murieron aproximadamente $\mathbf{1 7 , 0 0 0}$ personas (DITRA, 2012), hechos que llevaron a que los accidentes de tránsito se consolidaran como la segunda causa de muerte violenta en Colombia (Perdomo, 2010),

En términos generales, los accidentes de tránsito se deben a múltiples causas, entre las que se destacan: causas ambientales, causas externas (Gicquel, et-al, 2017), causas mecánicas y/o propias del vehículo (Thomas, Morris, Talbot, \& Fagerlind, 2013), además del factor humano (Petridou, Moustaki, 2000). Sin embargo, investigaciones coinciden en que el factor humano es el elemento de mayor incidencia en la ocurrencia de los accidentes (Montoro, Alonso, Esteban, \& Toledo, 2000; WHO, 2004), ya que, al fin y al cabo, el ser humano es quien dirige la conducción. Por lo anterior, estudiar los actores viales (sus características y sus estados en general) se hace imperante en la búsqueda de la reducción de fatalidades en la vía.
Tal estudio implica, entre tantas cosas, atender al estado de salud de los conductores, debido a las implicaciones (Razmara, et-al, 2018; Huang, et-al, 2017) que tiene la relación entre accidentes y salud. Específicamente, la literatura en torno a la seguridad vial sugiere que el estado de salud de los conductores puede afectar su desempeño en la tarea de conducir y que, a su vez, la conducción puede afectar el estado de salud (Alonso, et-al, 2008). Como variable, la salud comprende la salud física y la salud mental y, congruentemente, un estado de salud óptimo implica un equilibrio entre el cuerpo y la mente (WHO, 1948). Cada uno de estos elementos es estudiado de diferentes maneras y se materializa en la vida de diversas formas.

Específicamente dentro de la salud mental existen variables como la agresividad, el estrés y el bienestar psicológico que, al guardar relación con la accidentalidad, resultan de especial interés para el campo del tráfico. En cuanto a la agresividad, se han encontrado asociaciones entre historial de lesión cerebral traumática, mayores índices de agresividad al volantes y accidentes (llie, et-al, 2015). Al respecto, Ruiz, Beltrán, Gómez, Lamus y leal-Salazar (2014) encontraron relaciones directas entre niveles de ira y hostilidad y la disposición a violar normas de tránsito (sobre límites de velocidad y consumo de alcohol antes de conducir). Por su parte, Ruiz y López (2010) hallaron una correlación directa entre niveles de hostilidad y número de accidentes viales en los dos últimos años autoinformados. Las personas más vulnerables a la agresividad o ira de los conductores en la carretera son los peatones y ciclistas, quienes se constituyen como víctimas al resultar lesionados tras un accidente. Ellos tienen más probabilidad de sufrir, a largo plazo, consecuencias relacionadas con su salud física y mental (Cavacuiti, et-al, 2013). Es preciso mencionar que, si bien la agresividad en la conducción es un concepto muy estudiado, no se ha llegado a un consenso entre investigadores (Dula, \& Geller, 2003). 
El estrés es concebido como el cambio de un estado de calma a un estado de agitación, consecuencia de altas cargas laborales, de tensiones en la vida cotidiana y, en el campo del tráfico, consecuencia de los atascos/trancones, la velocidad o agresividad en la conducción, entre otros. Adicionalmente, el estrés guarda estrecha relación con aspectos de personalidad y con habilidades de afrontamiento, por lo que está ligado a constructos como el bienestar y las habilidades y dificultades percibidas en la conducción. Las investigaciones en torno al estrés son concluyentes frente a su relación con los accidentes y, en consonancia, es considerado uno de los factores incidentes más importantes (Magaña, et-al, 2017).

Pasando al bienestar, el Consejo Nacional para la Investigación de Estados Unidos lo considera un factor determinante en la calidad de vida de las personas y en su esperanza de vida (NRC, 2001), por ello es tan importante en lo que a salud pública se refiere. La Organización para la Cooperación y el Desarrollo Económicos (OCDE, 2013), recomienda que en el estudio del bienestar subjetivo (el bienestar percibido por la persona) sean tenidos en cuenta los datos sociodemográficos (como la edad, el género, el estado civil, el ingreso salarial, entre otros) los cuales dependerán de los objetivos planteados en los estudios. Dichos datos son esenciales para describir la población objeto de estudio con el fin de realizar análisis diferenciales. En el caso específico de estudios sobre seguridad en la carretera, variables como el historial de accidentes, tipo de conductor, experiencia en la conducción, edad, género, entre otras, son incluidas para el entendimiento del fenómeno (Mohtasham-Amiri, et-al, 2016; La Torre, et-al, 2007).

Existe evidencia que sostiene que éstas y otras variables afectan a las personas en mayor o menor grado, según su relación con la tarea de conducir. Por ejemplo, se ha encontrado que los conductores profesionales tienen más posibilidades de presentar peores índices de salud en comparación con los conductores particulares (por ejemplo, mayor probabilidad de desarrollar obesidad (Rosso, et-al, 2015)) y/o cáncer (Rafnsson, \& Gunnarsdóttir, 1991). Diferentes reportes concluyen que los conductores profesionales se encuentran en mayor riesgo en comparación con los conductores cuya actividad laboral no es la conducción, situación que resulta preocupante. También existe evidencia que, en Italia, al menos una cuarta parte de los conductores de camiones duermen dos horas menos de lo recomendado y que presentan somnolencia excesiva durante el día, hecho que se relaciona con más accidentes (Garbarino, et-al, 2016). Por último, en estudios sobre auto-percepción, los conductores profesionales se autodescriben como un grupo con estilos de vida poco saludables (Greenfield, et-al, 2016).

En torno a este tipo de temas, las publicaciones en español y sobre países Latinoamericanos sobre son más bien escasas -a excepción de las publicaciones en España-, hecho que contrasta con la cantidad de información disponible en otros contextos como el europeo o el estadounidense. Las experiencias en países de habla hispana se encuentran predominantemente influenciadas por lo reportado en países industrializados (Nazif, 2001), y no proveen respuestas sobre la accidentalidad en contextos latinoamericanos. Por lo anterior, una de las tareas que apremia en la investigación en salud ocupacional es el trabajo y la capacitación de investigadores en estos países, para así poder salvar la mayor cantidad de vidas (Felknor, et-al, 2006), otra es el desarrollo de instrumentos de medición en el campo del tráfico.

Teniendo en cuenta lo anterior, el presente trabajo estudia el fenómeno de la accidentalidad vial a partir del autoreporte de dos tipos de conductores colombianos, a saber: conductores profesionales y particulares. El objetivo principal es describir su situación social, su estado de salud, de bienestar psicológico, su nivel de estrés, de agresividad, así como las dificultades que presentan para conducir. Cinco hipótesis exploratorias son planteadas, en las que se espera los conductores profesionales (CPR) presenten índices menores en las evaluaciones de salud general, bienestar, estrés, agresividad y dificultades en la conducción, además de que presenten condiciones sociales menos favorables que aquellas de los conductores particulares (CPA). Tales hipótesis son:

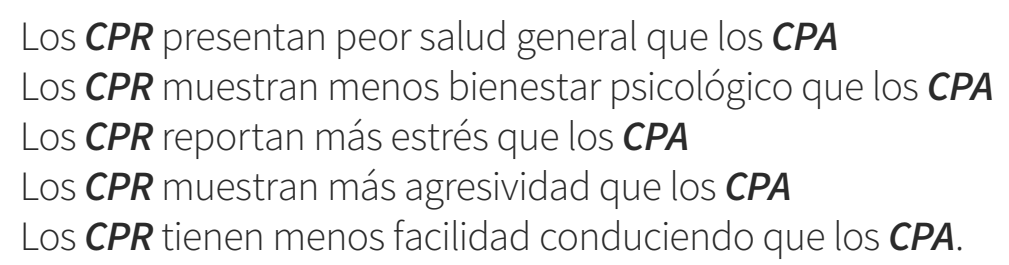

Estas hipótesis se basan en el hecho que los $C P R$ pasan más tiempo conduciendo, por lo que están más expuestos a los estresores de la vía y desarrollan más cansancio debido al mayor número de horas conduciendo.

\section{Método}

\section{Participantes}

Empresas bogotanas de transporte público, escuelas de enseñanza de conducción y centros de reconocimiento de conductores cooperaron participando en un estudio transversal llevado a cabo en el segundo semestre del año 2014 -la única condición para participar era ser conductor de cualquier tipo de vehículo-. Dicho estudio buscaba reunir información de 2,000 conductores colombianos y el espacio muestral fue escogido a conveniencia.

Para el mes de noviembre se contaba con la participación de $n=591$ participantes, de esta muestra preliminar, 250 conductores particulares (CPA) y $\mathbf{2 5 0}$ conductores profesionales (CPR) -todos bogotanos- fueron seleccionados al azar, obteniendo finalmente una muestra $n=500$ (413 hombres y $\mathbf{6 3}$ mujeres) para el presente estudio (con dos submuestras $n_{C P A}=250$ y $n_{C P R}=250$, como se mencionó anteriomente). Esta muestra es representativa de la ciudad de Bogotá, pues el movimiento de su parque automotor ${ }^{1}$ fue de $\mathbf{1 4 , 8 2 4}$ vehículos para el año 2013 (DANE, 2014), y si el tamaño mínimo de muestra es calculado con un nivel de confianza del $\mathbf{9 5 \%}$ y un error del $\mathbf{5 \%}$, debe ser igual a $\mathbf{3 7 7}$ participantes. Tomando en consideración lo anterior, con $n=500$ el margen de error es reducido a 4.33\% (DSII, 2014).

Es preciso aclarar que, en este estudio, al hablar de conductores profesionales se hará referencia a aquellas personas quienes utilizan un vehículo para desempeñar su actividad económica principal, (incluyendo taxistas, conductores de buses, etc.) y al hablar de conductor particular se hará referencia quienes hacen uso de un vehículo, pero no para desempeñar su actividad económica principal (e.g., personas quienes usan el vehículo para movilizarse desde y hacia su lugar de trabajo).

\section{Medidas}

Diferentes instrumentos fueron empleados en formato encuesta, su uso en el ámbito del tráfico, la seguridad vial y la salud, ha demostrado índices altos de confiabilidad (Useche, \& Ruiz, 2011) y sus versiones en

1 El parque automotor debe ser conducido por una persona con licencia de conducción vigente. 
español se han empleado en otros países (Díaz, et-al, 2006). Las variables objeto de estudio fueron: salud general, bienestar psicológico, estrés al conducir, agresión, y dificultades percibidas en la conducción, todas representadas en los instrumentos. El tiempo promedio de aplicación fue de 40 minutos por persona, y se utilizaron los puntajes globales del auto-reporte de los conductores.

\section{Instrumentos}

1. Cuestionario de información sociodemográfica y de accidentalidad. Considera el género, la edad, el nivel educativo, la situación laboral, el tipo de conductor, el ingreso salarial, su experiencia en conducción y el total de accidentes.

2. General Healht Questionnaire GHQ-12. Es utilizado en la detección de trastornos psiquiátricos en población general, y a través de 12 ítems indaga sobre el estado general de salud de la persona (mayores puntajes reflejan peor estado de salud general (Lobo, Pérez-Echeverría, \& Artal, 1986)). Es considerado válido para el estudio de población colombiana (Ruiz, García, Suárez, 2017), por lo que ha sido utilizado, en el país, en poblaciones de estudiantes universitarios bogotanos, una muestra de la población general y una muestra clínica, presentando un buen índice de consistencia interna ( $\alpha$ = 0.9). Un ejemplo de sus preguntas es: "Se ha sentido capaz de concentrarse en lo que está haciendo?”.

3. Psychological Well-Being Scale SPWB. Escala de $\mathbf{3 9}$ ítems que estudia diferentes dimensiones del bienestar psicológico, en la que valores altos indican más bienestar (Ryff, 1995, en van Dierendonck, 2004). Ha sido adaptada a población hispanohablante, obteniendo buenos índices de fiabilidad y en general buena consistencia interna entre las seis dimensiones teóricas que la componen -( $\alpha$ entre 0.83 y 0.68)- (Díaz, et-al, 2006). Algunas de sus preguntas son: "tengo clara la dirección y el objetivo de mi vida" y "siento que mis amistades me aportan muchas cosas".

4. Escala de Apreciación del Estrés en la Conducción EAE-C. Forma parte de un grupo de cuatro escalas cuyo objetivo es evaluar la incidencia de diversos hechos en la vida, específicamente ésta está diseñada para evaluar el estrés de las personas al conducir (mayor puntaje obtenido es interpretado como mayor estrés (Fernández Seara, \& Mielgo, 1992)). Ha sido utilizada en población colombiana, obteniendo coeficientes de alfa de Cronbach $\alpha=$ 0.92, (Useche, \& Ruiz, 2011). Algunas de las situaciones evaluadas son: la conducción lenta de otros conductores, los trancones y los viajes largos.

5. Aggression Questionnaire AQ. Cuestionario compuesto de $\mathbf{2 9}$ ítems, es uno de los auto-informes más utilizados para estudiar la agresividad (Ruiz, Arbeláez y Calderón, 2006). (puntuaciones altas refleja mayores niveles de agresividad (Buss, \& Perry, 1992)). Como instrumento es consistente entre lenguajes y muestras, y para la adaptación española tiene una congruencia de $\alpha=0.84$ (Vigil, Lorenzo, Codorniu, \& Morales, 2005). La persona debe responder si diferentes situaciones le caracterizan, por ejemplo: "Yo me involucro en peleas más que la mayoría de las personas".

6. Escala de Dificultades Percibidas en la conducción EDC. A través de $\mathbf{3 5}$ ítems, evalúa las posibles dificultades relacionadas con la conducción en cuatro dimensiones: norma (eg: respetar los semáforos en rojo), cultura ciudadana, dificultades específicas y estereotipos. Mayores puntajes reflejan mayor facilidad para conducir. En trabajos con población colombiana ha tenido una fiabilidad interna de entre ( $\alpha=0.88$ y 0.95), (Ruiz, \& López, 2010).

\section{Procedimiento}

Con el fin de realizar ajustes en el cuestionario, se realizó una prueba piloto del mismo con $n=57$ conductores. Una vez realizados los ajustes, diferentes empresas del sector de transporte público y privado y centros de reconocimiento de conductores -todos en Bogotá-fueron contactados al azar. La investigación contó con la participación de aquellas empresas y centros que manifestaron su interés en el estudio y fue llevada a cabo en las instalaciones de esas mismas empresas y centros, en una o varias sesiones dependiendo del espacio disponible.

Dos encuestadores con pleno conocimiento de las instrucciones de aplicación realizaron la recolección de información. El software estadístico de análisis de datos Stata $\mathbf{1 1 . 1}$ fue utilizado para llevar a cabo los análisis de datos: un análisis descriptivo fue realizada como primera medida y un análisis $\boldsymbol{T}$ Student para muestras independientes con $\boldsymbol{\alpha}=0.05$, para cada variable, fue realizado para comparar los dos tipos de conductores.

\section{Consideraciones éticas}

Los participantes firmaron un consentimiento informado dando cumplimiento a las disposiciones éticas de la Ley 1090 de 2006 y según el Código Deontológico y Bioético del Psicólogo. Mediante dicho consentimiento, los participantes fueron informados sobre los fines meramente académicos del estudio y la recolección de datos, sobre el carácter voluntario de su participación, sobre el respeto a su anonimato y sobre la confidencialidad respecto a sus datos personales y/o laborales.

\section{Resultados}

\section{Análisis descriptivo}

La muestra tenía una edad media años con una desviación estándar $S D=11.47$ años, el rango de edad iba desde los 16 hasta los 72 años y la mediana (p50), como medida más robusta, fue de 35 años. Cabe mencionar que esta variable tenía a una distribución asimétrica positiva (As = 0.48) y leptocúrtica $(C k=2.53)$ (Tabla 1 y Gráfico 1).

Tabla 1 Descriptivos variables sociodemográficas por tipo de conductor

\begin{tabular}{|c|c|c|c|c|c|c|c|c|c|}
\hline \multicolumn{2}{|c|}{ Tipo de Conductor } & $\mathrm{n}$ & $\ddot{X}$ & $S D$ & P50 & Mín. & Máx. & As & Ck \\
\hline \multirow{3}{*}{$\begin{array}{l}\text { Edad en } \\
\text { años }\end{array}$} & M. total & 463 & 37.40 & 11.48 & 35 & 16 & 72 & 0.49 & 2.54 \\
\hline & $C P R$ & 225 & 42.33 & 10.79 & 42 & 20 & 72 & -- & -- \\
\hline & $C P A$ & 238 & 32.68 & 10.06 & 31 & 16 & 71 & -- & -- \\
\hline \multirow{3}{*}{$\begin{array}{l}\text { Ingreso } \\
\text { salarial en } \\
\text { SMLV } 2014 \\
\text { Colombia }\end{array}$} & M. total & 307 & 2.86 & 2.66 & 2 & 1 & 33 & 6.13 & 60.47 \\
\hline & $C P R$ & 141 & 1.89 & 0.68 & 2 & 1 & 5 & -- & -- \\
\hline & $C P A$ & 166 & 3.68 & 3.36 & 3 & 1 & 33 & -- & -- \\
\hline \multirow{3}{*}{$\begin{array}{c}\text { Experiencia } \\
\text { al volante } \\
\text { en años }\end{array}$} & M. total & 473 & 15.40 & 15.40 & 14 & 0 & 52 & 0.64 & 2.64 \\
\hline & $C P R$ & 242 & 19.90 & 10.39 & 20 & 1 & 52 & -- & -- \\
\hline & $C P A$ & 231 & 10.68 & 9.59 & 8 & 0 & 48 & -- & -- \\
\hline \multirow{3}{*}{ Accidentes } & M. total & 370 & 0.93 & 1.53 & 0 & 0 & 10 & 2.58 & 12.51 \\
\hline & $C P R$ & 187 & 1.40 & 1.77 & 1 & 0 & 10 & -- & -- \\
\hline & $C P A$ & 183 & 0.45 & 1.03 & 0 & 0 & 5 & -- & \\
\hline
\end{tabular}




\section{Conductores profesionales y conductores particulares bogotanos}

Diagnóstico de condiciones sociodemográficas y bienestar general [análisis exploratorio]

Discriminando por tipo de conductor, se encontró que para CPR: $\ddot{X}=42$ años, $S D=10,7$ años, y p50 = 42; y para los CPA: $\ddot{X}=31$ años, $S D=10$ años, y p50 = 31 (ver Tabla 1). Según lo anterior, los CPA son personas más jóvenes en comparación con los CPR.

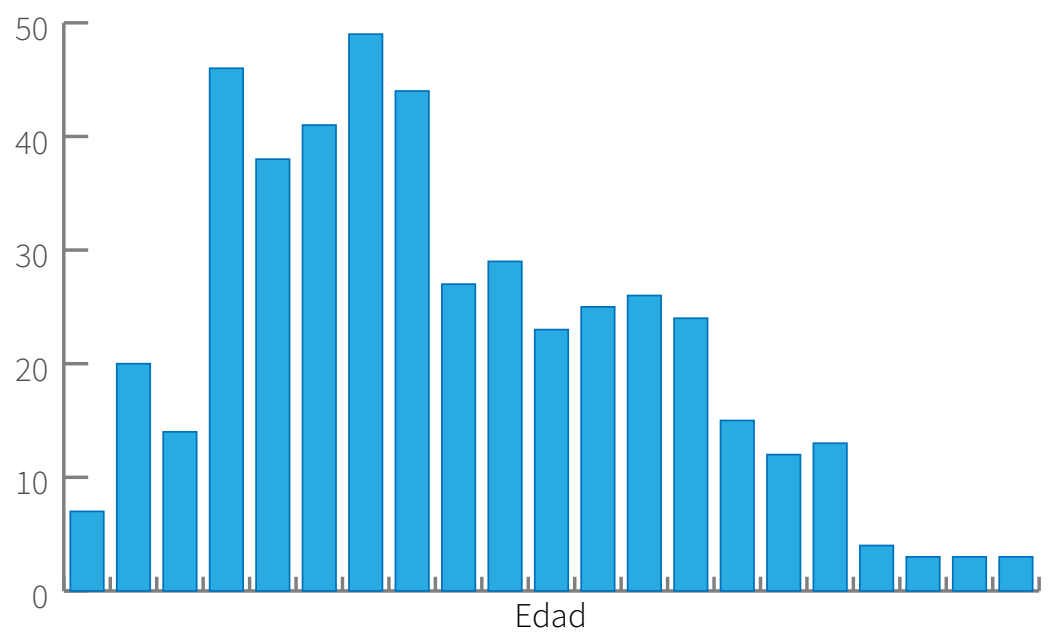

Gráfico 1 Histograma variable edad

En cuanto al nivel educativo, el $\mathbf{3 8 \%}$ de la muestra estaba en bachillerato, el $\mathbf{2 6 \%}$ era profesional, y el $\mathbf{1 5 \%}$ técnico; Discriminando por tipo de conductor, las mayores proporciones corresponden a: $\mathbf{C P R}$ con $\mathbf{6 3} \%$ (bachillerato) y CPA con $\mathbf{4 6 . 5 \%}$ (profesional) (Tabla 2).

Tabla 2 Frecuencias variable nivel educativo, por tipo de conductor

\begin{tabular}{|c|c|c|c|}
\hline Nivel educativo & $\begin{array}{l}\text { Conductor } \\
\text { profesional (CPR) }\end{array}$ & $\begin{array}{l}\text { Conductor particular } \\
\text { (CPA) }\end{array}$ & Total \\
\hline Sin estudios & 2 & 1 & 3 \\
\hline Primaria & 30 & 8 & 38 \\
\hline Bachillerato & 152 & 30 & 182 \\
\hline Técnico & 37 & 36 & 73 \\
\hline Tecnológico & 4 & 15 & 19 \\
\hline Profesional & 12 & 112 & 124 \\
\hline Especialización & 1 & 30 & 31 \\
\hline Maestría & 0 & 9 & 9 \\
\hline Total & 238 & 241 & 479 \\
\hline $\begin{array}{l}\text { Ingreso salarial } \\
\text { aproximado SMLV }\end{array}$ & $\begin{array}{c}\text { Conductor } \\
\text { profesional (CPR) }\end{array}$ & $\begin{array}{l}\text { Conductor particular } \\
\text { (CPA) }\end{array}$ & Total \\
\hline 1 & 35 & 21 & 56 \\
\hline $2-4$ & 104 & 117 & 211 \\
\hline $5-10$ & 2 & 34 & 36 \\
\hline $11-33$ & 0 & 4 & 4 \\
\hline Total & 141 & 166 & 307 \\
\hline
\end{tabular}

Fuente: Elaboración propia

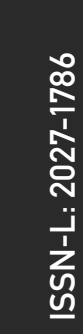

50

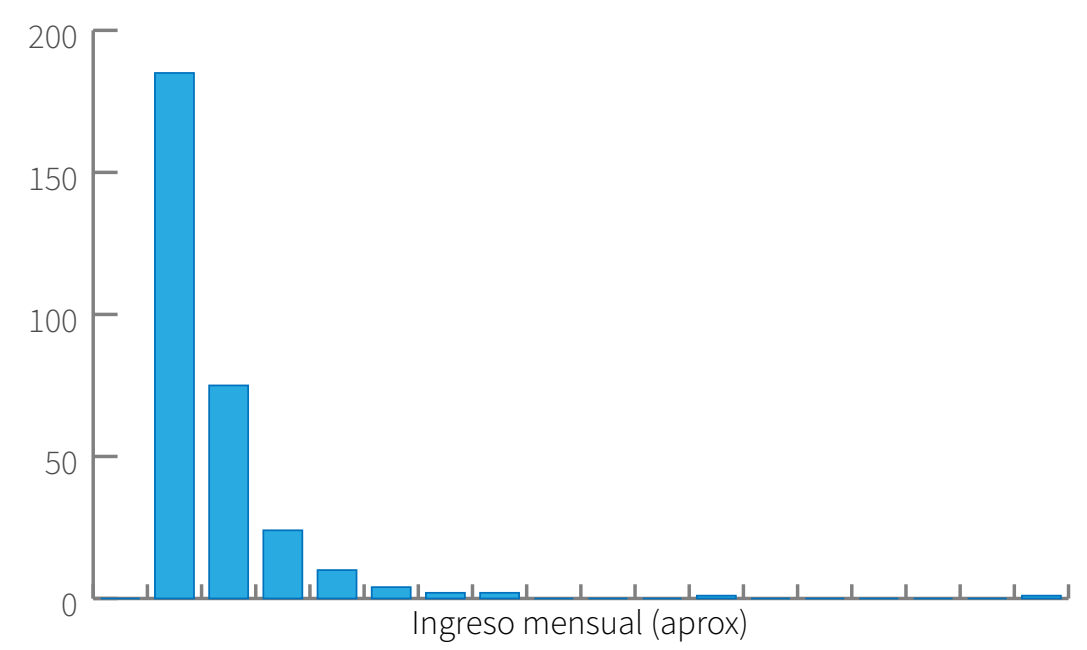

Gráfico 2 Histograma variable ingreso mensual aproximado

Respecto al ingreso económico mensual aproximado (calculado por salarios mínimos legales vigentes -SMLV- al 2014), se obtuvo $\ddot{X}=$ 2.9 SMLV, $S D=2.7 S M L V$. Esta variable presentó una forma asimétrica a la derecha y leptocúrtica, afectada por valores extremos (Gráfico 2). Discriminando por tipo de conductor, se obtuvo que para $C P R: \ddot{X}=1.9$ $S M L V, S D=0.7 S M L V$, y para $C P A: \ddot{X}=3.7 S M L V, S D=3.4 S M L V($ Tabla 1$)$.
De acuerdo con lo anterior, los CPA son personas que tienen ingresos salariales mayores, y una mayor variedad y cantidad de ingresos en comparación con los CPR (ver Tabla 2 y Gráfico 3).

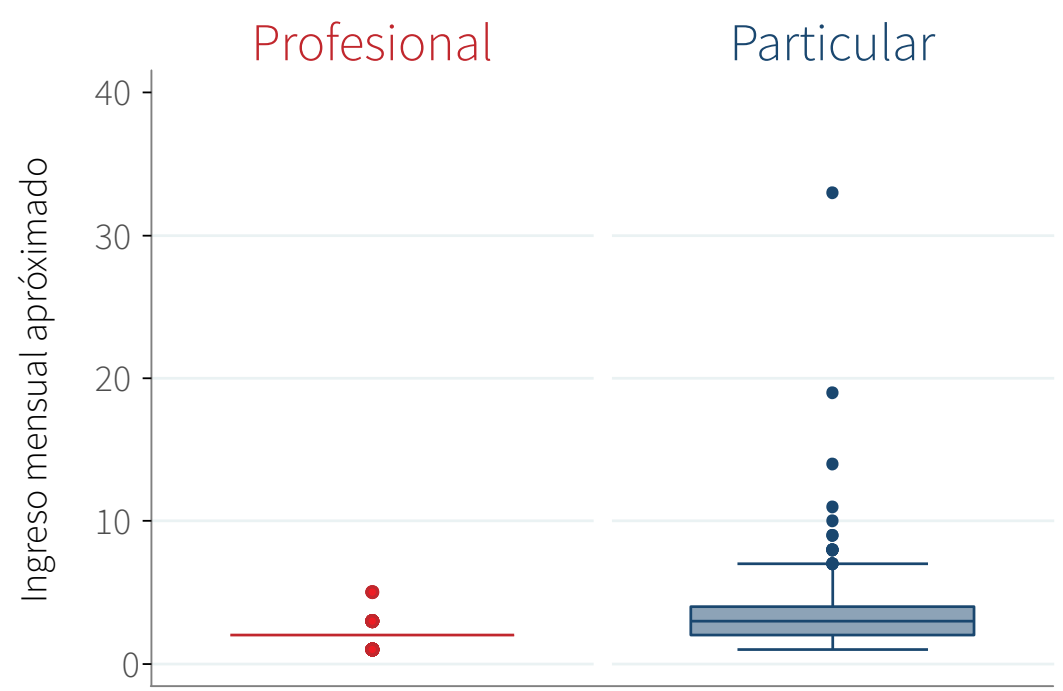

Gráfico 3 Box plot variable ingreso mensual aproximado por tipo de conductor

En cuanto al tiempo de experiencia (en años) al volante, se obtuvo $\ddot{X}=15.3$ años, $S D=11$ y p50 = 14. Discriminando por tipo de conductor, los CPA son personas con un promedio de menos años de experiencia en conducir, en comparación con los CPR (ver Tabla 1 y Gráfico 4).

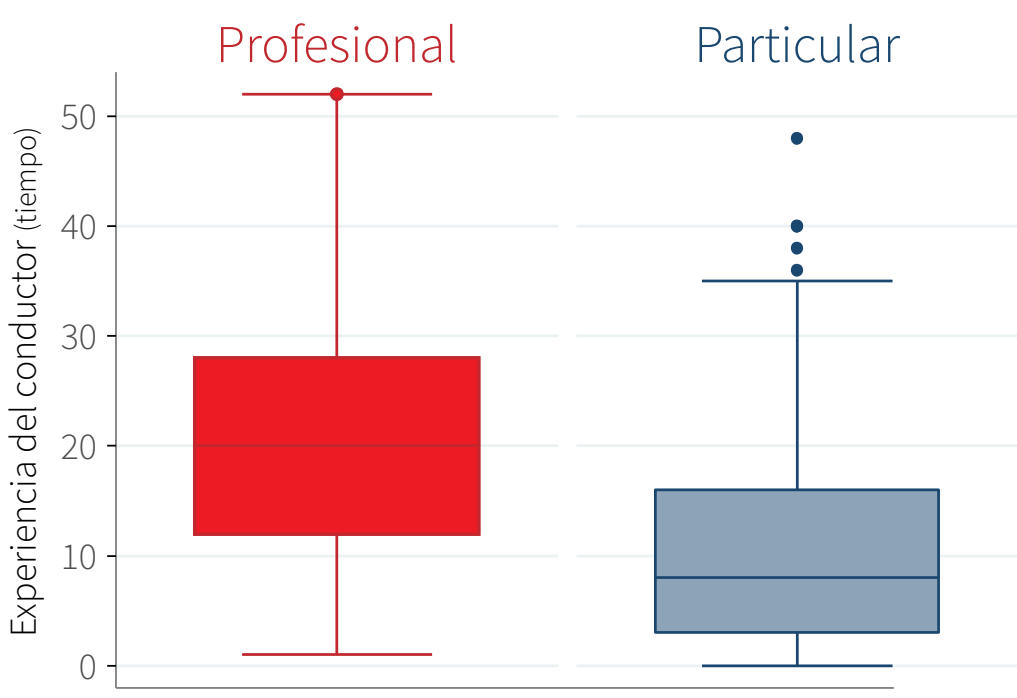

Gráfico 4 Box plot variable experiencia en conducción por tipo de conductor

Para la variable total de accidentes (durante el total de experiencia de conducción) se obtuvo $\ddot{X}=0.92, S D=1.95$, y p50 $=0$, y una distribución asimétrica positiva y leptocúrtica. En esta variable, los CPR obtuvieron $\ddot{X}=1.4, S D=1.7$, y p50 = 1; y los $C P A \ddot{X}=0.4$ años, SD $=1$, y p50 = 0. De lo anterior es posible afirmar que los $C P R$ reportan más accidentes de tránsito que los CPA (Tabla 1, Gráfico 5 y Gráfico 6).

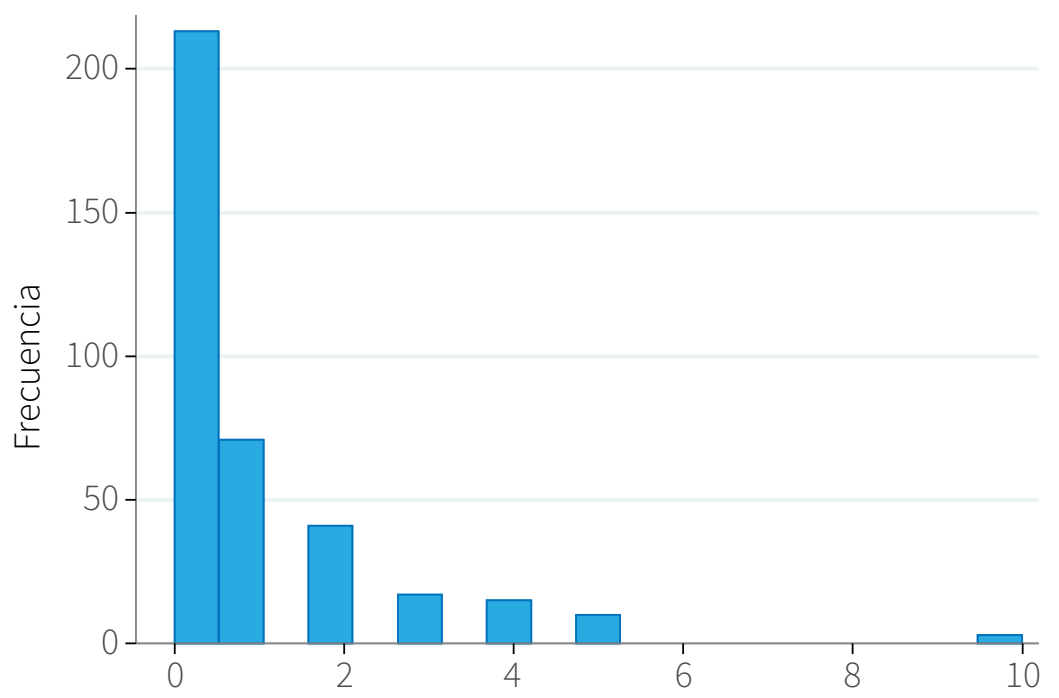

Gráfico 5 Histograma variable total de accidentes 


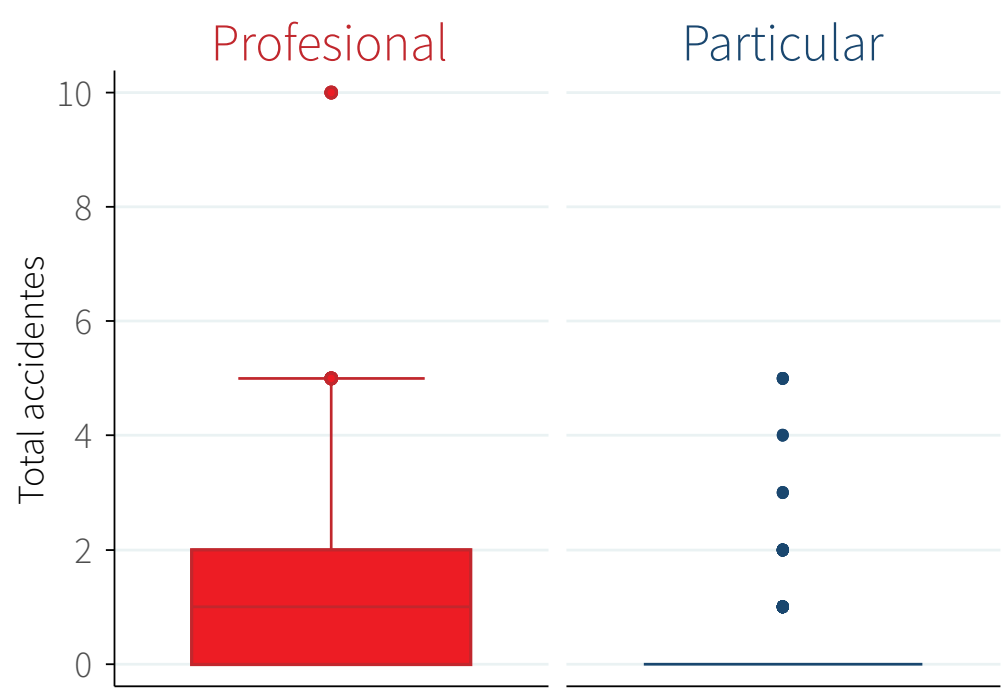

Gráfico 6 Box plot variable total de accidentes por tipo de conductor

Una prueba Chi $^{2}$ (ver Tabla 3) fue llevada a cabo como segundo paso para comparar las proporciones en cuanto al nivel educativo de ambos tipos de conductores. Según los resultados obtenidos, el nivel educativo sí es significativo y debe ser tomado en cuenta para el estudio de los tipos de conductor.

Tabla $3 \mathrm{Chi}^{2}$ para variable nivel educativo por tipo de conductor

\begin{tabular}{|c|c|c|c|c|c|c|c|c|c|c|}
\hline \multicolumn{2}{|c|}{$\begin{array}{c}\text { Tipo } \\
\text { Conductor }\end{array}$} & SE & BP & BM & TC & TN & PR & ES & MA & Total \\
\hline \multirow{3}{*}{$C P R$} & $\mathrm{Fr}$ & 2 & 30 & 152 & 37 & 4 & 12 & 1 & 0 & 238 \\
\hline & Fre & 1.5 & 18.9 & 90.4 & 36.3 & 9.4 & 15.4 & 15.4 & 4.5 & 238 \\
\hline & $\%$ & 0.42 & 6.26 & 31.73 & 7.72 & 0.84 & 0.21 & 0.21 & 0 & 49.69 \\
\hline \multirow{3}{*}{$C P A$} & $\mathrm{Fr}$ & 1 & 8 & 30 & 36 & 15 & 112 & 30 & 9 & 241 \\
\hline & Fre & 1.5 & 19.1 & 91.6 & 36.7 & 9.6 & 62.4 & 15.6 & 4.5 & 241 \\
\hline & $\%$ & 0.21 & 1.67 & 6.26 & 7.52 & 3.13 & 23.38 & 6.26 & 1.88 & 50.31 \\
\hline \multirow{3}{*}{ Total } & $\mathrm{Fr}$ & 3 & 38 & 182 & 73 & 19 & 124 & 31 & 9 & 479 \\
\hline & Fre & 3 & 38 & 182 & 73 & 19 & 124 & 31 & 9 & 479 \\
\hline & $\%$ & 0.63 & 7.93 & 38 & 15.24 & 3.97 & 25.89 & 6.47 & 1.88 & 100 \\
\hline
\end{tabular}

SE: Sin Estudios | EB: B. Primaria | BM: Bachiller | TC: Técnico | TN: Tecnológico | PR: Profesional | SP: Especialización | MA: Maestría Pearson chi 2(7) =217,9965 $\operatorname{Pr}=0,000 ;$ Fr: frecuencia, Fre: frecuencia esperada. Fuente: Elaboración propia

\section{Diferencia de medias}

Diferencias de medias se realizaron para comparar los dos grupos de conductores en las siguientes variables: salud (GHQ), bienestar psicológico (SPWB), estrés (EAE-C), agresividad (AQ), y dificultades en la conducción (EDC). Se plantearon $\mathbf{5}$ pruebas de hipótesis teniendo en cuenta la interpretación del puntaje global de cada cuestionario y/o variable. Para estudiar estas hipótesis se utilizó una prueba T Student para muestras independientes, con un nivel de confianza del 95\%.

Los resultados, tras la aplicación del GHQ-12, revelaron que los CPA estaban presentando peores niveles de salud en comparación con los CPR. De acuerdo con los resultados arrojados por la SPWB sobre el bienestar, no hubo evidencia estadística significativa para apoyar , no se rechazó la hipótesis nula (). Entonces, los CPA estaban presentando niveles menores de bienestar psicológico y obtuvieron puntajes de estrés más altos, en comparación con los CPR (Tabla 4).

Respecto a la agresividad, no hubo evidencia estadística significativa para considerar que los $C P R$ mostraban niveles de agresividad que CPA. Finalmente, respecto a las dificultades percibidas al conducir, los CPA estaban presentando menos facilidad para conducir que los CPR (ver Tabla 4).
Tabla 4 T-student para variables salud, bienestar y estrés, en dos muestras de conductores

\begin{tabular}{|c|c|c|c|c|c|c|}
\hline \multirow{2}{*}{$\begin{array}{c}\mathrm{GHQ} \\
\boldsymbol{\alpha}=0.764\end{array}$} & \multirow{2}{*}{$n$} & \multirow{2}{*}{$\ddot{x}$} & \multirow{2}{*}{ SE } & \multirow{2}{*}{$S D$} & \multicolumn{2}{|l|}{$\mathrm{Cl} 95 \%$} \\
\hline & & & & & LL & UL \\
\hline $\begin{array}{r}\text { Conductor profesional } \\
\qquad(C P R)\end{array}$ & 250 & 16.18 & 0.31 & 4.92 & 15.56 & 16.79 \\
\hline $\begin{array}{r}\text { Conductor particular } \\
(C P A)\end{array}$ & 250 & 19.45 & 0.30 & 4.74 & 18.86 & 20.04 \\
\hline Combinado & 500 & 17.81 & 0.23 & 5.09 & 17.36 & 18.26 \\
\hline \multicolumn{2}{|l|}{ Diff } & -3.27 & \multicolumn{2}{|l|}{0.43} & -4.12 & -2.42 \\
\hline \multicolumn{5}{|c|}{ Diff $=$ media $(C P R)-$ media $(C P A)$} & Cohen's d =-0.68 & $t=-7.5751$ \\
\hline \multicolumn{5}{|l|}{ Ho: diff = 0} & & $\mathrm{GL}=498$ \\
\hline \multicolumn{2}{|l|}{ Ha: diff<0 } & \multicolumn{3}{|c|}{ Ha: diff! = 0} & \multicolumn{2}{|c|}{ Ha: diff $>0$} \\
\hline \multicolumn{2}{|l|}{$\operatorname{Pr}(T<t)=0.0000$} & \multicolumn{3}{|c|}{$\operatorname{Pr}(|T|>|t|)=0.0000$} & \multicolumn{2}{|c|}{$\operatorname{Pr}(T>t)=1.0000$} \\
\hline $\begin{array}{c}\text { SPWB } \\
\boldsymbol{\alpha}=0.868\end{array}$ & $\mathrm{n}$ & $\ddot{x}$ & SE & SD & \multicolumn{2}{|l|}{ Cl $95 \%$} \\
\hline $\begin{array}{r}\text { Conductor profesional } \\
\qquad(C P R)\end{array}$ & 250 & 167.99 & 2.80 & 44.26 & 162.48 & 173.50 \\
\hline $\begin{array}{l}\text { Conductor particular } \\
\qquad(C P A)\end{array}$ & 250 & 159.22 & 2.74 & 43.39 & 153.82 & 164.63 \\
\hline Combinado & 500 & 163.61 & 1.97 & 44.00 & 159.74 & 167.47 \\
\hline \multicolumn{2}{|l|}{ Diff } & 8.76 & 3.92 & & 1.06 & 16.47 \\
\hline \multicolumn{2}{|c|}{ Diff = media $(\mathbf{C P R})-$ media $(\mathbf{C P A})$} & & & & Cohen's $d=0.18$ & $t=-2.2357$ \\
\hline \multicolumn{2}{|l|}{ Ho: diff = 0} & & & & & $\mathrm{GL}=498$ \\
\hline \multicolumn{2}{|l|}{ Ha: $\operatorname{diff}<0$} & \multicolumn{3}{|c|}{ Ha: diff! = 0} & \multicolumn{2}{|c|}{ Ha: diff>0 } \\
\hline \multicolumn{2}{|l|}{$\operatorname{Pr}(T<t)=0.9871$} & \multicolumn{3}{|c|}{$\operatorname{Pr}(|T|>|t|)=0.0258$} & \multicolumn{2}{|c|}{$\operatorname{Pr}(T>t)=0.0129$} \\
\hline $\begin{array}{c}\text { EAE-C } \\
\boldsymbol{\alpha}=0.941\end{array}$ & $n$ & $\ddot{x}$ & SE & $S D$ & Cl $95 \%$ & UL \\
\hline $\begin{array}{r}\text { Conductor profesional } \\
\qquad(C P R)\end{array}$ & 250 & 23.45 & 0.97 & 15.35 & 21.54 & 25.36 \\
\hline $\begin{array}{l}\text { Conductor particular } \\
\qquad(C P A)\end{array}$ & 250 & 27.08 & 0.99 & 15.69 & 25.12 & 29.03 \\
\hline Combinado & 500 & 25.26 & 0.70 & 15.61 & 23.89 & 26.64 \\
\hline Diff & & -3.62 & 1.39 & & -6.35 & -0.90 \\
\hline Diff $=$ media $(\boldsymbol{C P R})-$ media & (CPA) & & & & Cohen's $d=-0.27$ & $t=-2.6109$ \\
\hline Ho: $\operatorname{diff}=0$ & & & & & & $\mathrm{GL}=498$ \\
\hline Ha: $\operatorname{diff}<0$ & & & diff! = & & Ha: diff> & \\
\hline $\operatorname{Pr}(T<t)=0.0047$ & & $\operatorname{Pr}(|T|=$ & $|t|)=($ & 0.0093 & $\operatorname{Pr}(T>t)=0$. & .9953 \\
\hline
\end{tabular}

Nota: $\mathrm{Cl}=$ intervalo de confianza; LL: límite inferior; UL: límite superior; diff: diferencia; GL: Grados de libertad. Fuente: Elaboración propia

\section{Discusión}

Desde una perspectiva exploratoria, este estudio tuvo como objetivo principal profundizar en el conocimiento de las características y condiciones propias de dos tipos de conductores: profesionales y particulares. Tendencias interesantes fueron halladas para cada una de las poblaciones, además de diferencias significativas en cuanto al estado de salud autoreportado por los participantes.

Principalmente, los resultados sugieren que existen diferencias importantes en las características sociales y económicas de los conductores, las variables descriptoras de los rasgos de cada población difieren bastante. En general, los conductores profesionales (CPR) son mayores, tienen más años de experiencia al conducir, tienen ingresos salariales inferiores, menores niveles educativos, mayor exposición a la conducción y mayor número de accidentes reportados, todo esto en comparación con los conductores particulares (CPA). Dichos aspectos han sido relacionados con peores índices de salud y bienestar, lo que 
guarda congruencia con el hecho que los conductores profesionales (CPR) hayan sido identificados con anterioridad como un grupo de riesgo precisamente por este tipo de características (Backman, 1983; Ronna, et-al, 2016). Cabe mencionar que se ha demostrado que la cantidad de horas conducidas, el sueño y la conducción a tempranas horas de la mañana, pueden aumentar el riesgo de accidentes (Bougard, et-al, 2012).

Siguiendo con las diferencias, los conductores particulares (CPA) y profesionales (CPR) presentan unas significativas en cuanto al nivel de escolaridad, pues últimos (CPR) tienen índices de escolarización más bajos que su contra parte. El nivel de escolaridad es de suma importancia debido a la relación entre peores índices de salud y nivel educativo (Baker, et-al, 2011). Además, esta variable también muestra resultados significativos en correlación con accidentes de tráfico (Sami, et-al, 2013), tendencia que se repite para la variable ingreso salarial (Lynch, et-al, 2004).

En términos generales, los conductores profesionales (CPR) en Bogotá presentanunaseriedecaracterísticas demográficasusualmente asociadas con mayor probabilidad de sufrir accidentes de tráfico. Sin embargo, los resultados del presente trabajo muestran tendencias en esta población, para las variables alud y bienestar, significativamente diferentes a lo esperado. En efecto, son los conductores particulares (CPA) quienes están reportando peores índices de salud, menores índices de bienestar, mayores puntajes en estrés y menos facilidad para conducir -no hay resultados significativos para la agresión. Esto muestra que el fenómeno de la accidentalidad vial se comporta de manera diferente a lo encontrado y reportado en la literatura de otros países (Öz, Özkan, \& Lajunen, 2010), hecho que obliga a continuar investigando sobre este tema en nuestro país y que resalta la necesidad de análisis diferenciales por actores viales.

Tabla 5 t-Student para variables agresión y dificultades en la conducción, en dos muestras de conductores

\begin{tabular}{|ccccccc|}
$\begin{array}{c}\text { AQ } \\
\alpha=0.919\end{array}$ & $n$ & $\ddot{X}$ & SE & SD & LL & UL \\
\hline $\begin{array}{r}\text { Conductor profesional } \\
\text { (CPR) }\end{array}$ & 250 & 43.54 & 1.15 & 18.11 & 41.28 & 45.79 \\
$\begin{array}{r}\text { Conductor particular } \\
\text { (CPA) }\end{array}$ & 250 & 45.53 & 1.20 & 18.97 & 43.17 & 47.89 \\
Combinado & 500 & 44.53 & 0.83 & 18.55 & 42.90 & 46.16 \\
Diff & & -2.00 & 1.66 & & -5.25 & 1.26 \\
\hline
\end{tabular}

Diff = media $(\mathbf{C P R})-$ media

$(C P A)$

Cohen's $d=-0.11 \quad t=-1.2034$
Ho: diff $=0$

Ha: diff $<0$

$\operatorname{Pr}(T<\mathrm{t})=0.1147$

\begin{tabular}{|c|c|c|c|c|c|c|}
\hline \multirow{2}{*}{$\begin{array}{c}\text { EDC } \\
\alpha=0.963\end{array}$} & \multirow{2}{*}{$\mathrm{n}$} & \multirow{2}{*}{$\ddot{x}$} & \multirow{2}{*}{ SE } & \multirow{2}{*}{ SD } & \multicolumn{2}{|c|}{$\mathrm{Cl} 95 \%$} \\
\hline & & & & & LL & UL \\
\hline $\begin{array}{r}\text { Conductor profesional } \\
(C P R)\end{array}$ & 250 & 141.04 & 1.97 & 31.20 & 137.16 & 144.93 \\
\hline $\begin{array}{r}\text { Conductor particular } \\
(\text { (CPA) }\end{array}$ & 250 & 132.61 & 2.19 & 34.70 & 128.29 & 136.93 \\
\hline Combinado & 500 & 136.83 & 1.49 & 33.23 & 133.91 & 139.75 \\
\hline Diff & & 8.43 & 2.95 & & 2.63 & 14.23 \\
\hline
\end{tabular}

Diff $=$ media $(\mathbf{C P R})-$ media

(CPA)

Cohen's $d=0.28 \quad t=2.8569$

Ho: diff $=0$

Ha: diff $<0$

$\operatorname{Pr}(T<\mathrm{t})=0.9978$

Ha: diff $!=0$

$G L=498$

Ha: diff $>0$

$\operatorname{Pr}(|T|>|t|)=0.0045$

$\operatorname{Pr}(T>t)=0.0022$
Nota: $\mathrm{Cl}=$ intervalo de confianza; LL: límite inferior; UL: límite superior; diff: diferencia; GL: Grados de libertad. Fuente: Elaboración propia

\section{Conclusiones}

A partir de este trabajo es posible concluir que existe una necesidad imperante de estudiar, de manera diferencial, las poblaciones y subpoblaciones dentro de la amplia categoría de actores viales, especialmente los conductores profesionales y particulares.

La conducción profesional en Bogotá implica una mayor exposición a diferentes elementos de riesgo en torno al tema de la movilidad y a las variables relacionadas con el status socioeconómico, en particular menor ingreso socioeconómico, en comparación con otros grupos poblaciones adultos.

Es de destacar que, a pesar que se esperaba que los conductores profesionales (CPR) presentaran peor salud, menor bienestar psicológico, y mayor estrés, los hallazgos sugieren que son los conductores particulares (CPA) quienes presentan peores indicadores autoreportados de salud. Los conductores profesionales (CPR) en efecto presentan mayores índices de accidentalidad que los conductores particulares (CPA), por lo cual las relaciones entre estrés en la conducción y accidentalidad parecen ser más complejas de lo que ha mostrado la literatura hasta el momento.

Por ende, más estudios deben ser realizados, que indaguen por qué los conductores particulares (CPA) reportan índices más bajos en salud y bienestar. Y que revelen el papel que juegan las variables subyacentes a los factores sociodemográficos y los elementos asociados a las mismas, como factor protector o de riesgo en la población de conductores. Adicionalmente, estudio a profundidad sobre las escalas utilizadas debe llevarse a cabo, o un estudio sobre el diseño de escalas más apropiadas para la situación verdadera de movilidad en Colombia. Lo anterior en tanto los resultados sugieren que la población de conductores profesionales (CPR) colombianos presentan condiciones de salud y bienestar diferentes a lo que dicta la literatura, y debe estudiarse si lo anterior se debe a un sesgo de deseabilidad social, o a que las escalas no son las adecuadas para el estudio de esta realidad (a pesar, aunque presenten buenos índices de fiabilidad por alfa de Cronbach).

Los conductores profesionales (CPR) pudieron haber alterado sus respuestas en las diferentes escalas debido al temor a que los resultados en la batería fueran conocidos por sus empleadores, aunque en el momento de la aplicación de la encuesta se les indicó que los datos sólo serían analizados en conjunto (todos los sujetos a la vez) por el equipo académico que diseñó y aplicó la batería, y que los datos individuales no serían entregados a las empresas de transporte. Siguiendo esta línea, el papel de la deseabilidad social aparece como una variable a considerar en futuros estudios, junto a la de una posible habituación a estresores viales debido a la mayor cantidad de horas que pasan los conductores en las vías.

\section{Limitaciones y futuras investigaciones}

Resultó sorprendente el hecho que los conductores profesionales (CPR) presentaran mejores índices de salud y bienestar en general, sin embargo, estos podrían explicarse por limitaciones relacionadas con las escalas de autoreporte: 1) los instrumentos pueden no ser los correctos para esta población, comprometiendo la validez de constructo; 2) los participantes asociados a empresas pueden sentirse inseguros al responder libremente las preguntas. Aunque un 
consentimiento informado haya sido empleado, la deseabilidad social puede influir en las respuestas de los participantes quienes buscan mantener su estabilidad laboral.

Por otro lado, los análisis realizados no permiten llevar a cabo inferencias o predicciones sobre la accidentalidad, por ello, los mismos datos deben ser tratados por medio de otros análisis (como análisis factorial para cada una de las escalas, que permita conocer la estructura de estos instrumentos), además de complementar con observaciones y estudios longitudinales, con los cuales triangular los hallazgos, lo que a su vez permitiría realizar otro tipo de análisis y llegar, quizá, a otro tipo conclusiones.

Finalmente, aunque no se encontraron resultados significativos en la variable de agresividad, debe considerarse que ésta fue evaluada con la Escala $A Q$, que tiene cuatro dimensiones que a su vez deberían analizarse en próximas investigaciones: la ira y/o la hostilidad, variables asociadas con la accidentalidad, pero no la agresividad verbal ni física. Es posible que estas diferencias no puedan ser percibidas como significativas con el puntaje global del $\mathbf{A Q}$.

\section{Financiamiento}

Este estudio fue llevado a cabo gracias a la beca Jóvenes Investigadores del Departamento Administrativo de Ciencia, Tecnología e Innovación (Colciencias), otorgada al grupo Procesos y Métodos en Psicología Jurídica, del Laboratorio de Psicología Jurídica, del Departamento de Psicología de la Universidad Nacional de Colombia (Sede Bogotá). Los resultados completos de la muestra final pueden ser consultados en Serge (2015).

\section{Agradecimientos}

Manifestamos agradecimiento a las empresas, centros de reconocimiento $\mathrm{y}$, en general, a los participantes que colaboraron en este estudio. También al equipo de investigación que, en alguna medida, participó en el diseño, recolección y sistematización de la información.

\section{Referencias}

Alonso, F., Esteban, C., Calatayud, C., Alamar, B., \& Egido, A. (2008). Salud vial. Teoría y prácticas de los trastornos físicos y psíquicos en la conducción. Valencia, Cuadernos de reflexiones Attitudes.

Backman, A. L. (1983). Health survey of professional drivers. Scand J Work Environ Health, 9(1), 30-35.

Baker, D. P., Leon, J., Smith Greenaway, E. G., Collins, J., \& Movit, M. (2011). The Education Effect on Population Health: A Reassessment. Population and Development Review, 37(2), 307-332. doi:10.1111/j.1728-4457.2011.00412.x

Bougard, C., Espie, S., Larnaudie, B., Moussay, S., \& Davenne, D. (2012). Effects of time of day and sleep deprivation on motorcycle-driving performance. PLoS One, 7(6), e39735. doi:10.1371/journal.pone.0039735

Buss, A., \& Perry, M. (1992). The Aggression Questionnaire. Journal of Personality and Social Psychology, 63, 452-459.

Cavacuiti, C., Ala-Leppilampi, K. J., Mann, R. E., Govoni, R., Stoduto, G., Smart, R., \& Locke, J. A. (2013). Victims of Road Rage: A Qualitative Study of the Experiences of Motorists and Vulnerable Road Users. Violence and Victims, 28(6), 1068-1084. doi:10.1891/0886-6708.vv-d-12-00068
DANE (2014). Anexo 1. Movimiento del parque urbano automotor y pasajeros transportados, según áreas metropolitanas y ciudades.

Diaz, D., Rodriguez-Carvajal, R., Blanco Abarca, A., Moreno-Jimenez, B., Gallardo, I., Dirk, C. V. Y., \& Dierendonck, D. (2006). Spanish adaptation of the Psychological Well-Being Scales (PWBS). Psicothema, 18(3), 572-577.

DITRA (2012). Cifras de accidentalidad vial en Colombia: Años 2009 a 2011. Documento institucional. División de Tránsito y Transporte. Bogotá: Policía Nacional de Colombia.

DSII, Departamento de Sistemas Informáticos Integrales. (2014). Calculadora para obtener tamaño de una muestra. Facultad de Medicina, Universidad Nacional del Nordeste. Obtenido de: http://www.med.unne.edu.ar/ biblioteca/calculos/calculadora.htm

Dula, C. S., \& Geller, E. S. (2003). Risky, aggressive, or emotional driving: addressing the need for consistent communication in research. J Safety Res, 34(5), 559-566.

Felknor, S. A., Delclos, G. L., Gimeno, D., Wesseling, I., Monge, P., Chavez, J., . Schulze, L. J. (2006). Funding of pilot projects in Latin America: a tool for capacity building in occupational health research. Int J Occup Environ Health, 12(4), 408-414. doi:10.1179/oeh.2006.12.4.408

Fernández Seara, J., \& Mielgo, N. (1992). Escalas de apreciación del estrés (EAE). Publicaciones de psicología Aplicada. Madrid: TEA.

Garbarino, S., Durando, P., Guglielmi, O., Dini, G., Bersi, F., Fornarino, S., Magnavita, N. (2016). Sleep Apnea, Sleep Debt and Daytime Sleepiness Are Independently Associated with Road Accidents. A Cross-Sectional Study on Truck Drivers. PLOS ONE, 11(11), e0166262. doi:10.1371/journal. pone. 0166262

Gicquel, L., Ordonneau, P., Blot, E., Toillon, C., Ingrand, P., \& Romo, L. (2017). Description of Various Factors Contributing to Traffic Accidents in Youth and Measures Proposed to Alleviate Recurrence. Frontiers in Psychiatry, 8, 94. doi:10.3389/fpsyt.2017.00094

Gopalakrishnan, S. (2012). A Public Health Perspective of Road Traffic Accidents. Journal of Family Medicine and Primary Care, 1(2), 144-150. doi: $10.4103 / 2249-4863.104987$

Greenfield, R., Busink, E., Wong, C. P., Riboli-Sasco, E., Greenfield, G., Majeed, A., ... Wark, P. A. (2016). Truck drivers' perceptions on wearable devices and health promotion: a qualitative study. BMC Public Health, 16, 677. doi:10.1186/s12889-016-3323-3

Huang, H. L., Yin, Q. Y., Schwebel, D. C., Ning, P. S., \& Hu, G. Q. (2017). Availability and consistency of health and non-health data for road traffic fatality: Analysis of data from 195 countries, 1985-2013. Accident Analysis and Prevention, 108, 220-226. doi:10.1016/j.aap.2017.08.033

Ilie, G., Mann, R. E., Ialomiteanu, A., Adlaf, E. M., Hamilton, H., Wickens, C. M., . Cusimano, M. D. (2015). Traumatic brain injury, driver aggression and motor vehicle collisions in Canadian adults. Accident Analysis and Prevention, 81, 1-7. doi:10.1016/j.aap.2015.04.021

La Torre, G., Van Beeck, E., Quaranta, G., Mannocci, A., \& Ricciardi, W. (2007) Determinants of within-country variation in traffic accident mortality in Italy: a geographical analysis. International Journal of Health Geographics, 6, 49. doi:10.1186/1476-072X-6-49

Ley 1090 (2006). Por la cual se reglamenta el ejercicio de la profesión de Psicología, se dicta el Código Deontológico y Bioético y otras disposiciones. Colombia.

Lobo, A., Pérez-Echevarria, M. J. y Artal, J. (1986). Validity of the scaled version of the General Health Questionnaire (GHQ-28) in a Spanish population. Psychological Medicine, 16, 135-140.

Lynch, J., Smith, G. D., Harper, S., Hillemeier, M., Ross, N., Kaplan, G. A., \& Wolfson, M. (2004). Is Income Inequality a Determinant of Population Health? Part 1. A Systematic Review. The Milbank Quarterly, 82(1), 5-99. doi:10.1111/j.0887378X.2004.00302.X

Magaña, V. C., Organero, M. M., Alvarez-Garcia, J. A., \& Rodriguez, J. Y. F. (2017). Design of a Speed Assistant to Minimize the Driver Stress. Adcaij-Advances in Distributed Computing and Artificial Intelligence Journal, 6(3), 45-56. doi:10.14201/adcaij2017634556

Mcllvenny, S. (2006). Road Traffic Accidents - A Challenging Epidemic. Sultan Qaboos University Medical Journal, 6(1), 3-5.

Mohtasham-Amiri, Z., Dastgiri, S., Davoudi-kiakalyeh, A., Imani, A., \& Mollarahimi, K. (2016). An Epidemiological Study of Road Traffic Accidents in Guilan Province, Northern Iran in 2012. Bulletin of Emergency, \& Trauma, 4(4), 230-235.

Montoro, L., Alonso, T., Esteban, C., \& Toledo, F. (2000). Manual de seguridad vial: El factor humano. Barcelona: Ariel -INTRAS. 


\section{Conductores profesionales y conductores particulares bogotanos}

\section{Diagnóstico de condiciones sociodemográficas y bienestar general [análisis exploratorio]}

Nazif, J. I. (2011). Testing and Contrasting Road Safety Education, Deterrence, and Social Capital Theories: A Sociological Approach to the Understanding of Male Drink-Driving in Chile's Metropolitan Region. Annals of Advances in Automotive Medicine / Annual Scientific Conference, 55, 313-323.

NRC (2001). National Research Council (US) Panel on a Research Agenda and New Data for an Aging World. Preparing for an Aging World: The Case for CrossNational Research. Washington (DC): National Academies Press (US); 2001. 7, Well-Being: Concepts and Measures. Obtenido de: https://www.ncbi.nlm. nih.gov/books/NBK98384/

OECD, (2013). Organisation for Economic Co-operation and Development (OECD). OECD Guidelines on Measuring Subjective Well-being. Paris: OECD Publishing; 2013 Mar 20. 3, Measuring subjective well-being. Obtenido de: https://www.ncbi.nIm.nih.gov/books/NBK189567/

Öz, B., Özkan, T., \& Lajunen, T. (2010). An investigation of the relationship between organizational climate and professional drivers' driver behaviours. Safety science, 48(10), 1484-1489.

Perdomo, M. (2010). Lesiones en accidentes de tránsito. Colombia, 2010. Un solo evento con incalculables consecuencias. Forensis, datos para la vida. Bogotá: Instituto Nacional de Medicina Legal y Ciencias Forenses, INMLCF.

Petridou, E., \& Moustaki, M. (2000). Human factors in the causation of road traffic crashes. Eur J Epidemiol, 16(9), 819-826.

Rafnsson, V., \& Gunnarsdottir, H. (1991). Mortality among professional drivers. Scand J Work Environ Health, 17(5), 312-317.

Razmara, A., Aghamolaei, T., Madani, A., Hosseini, Z., \& Zare, S. (2018). Prediction of safe driving Behaviours based on health belief model: the case of taxi drivers in Bandar Abbas, Iran. BMC public health, 18(1), 380. doi:10.1186/ s12889-018-5300-5

Ronna, B. B., Thiese, M. S., Ott, U., Effiong, A., Murtaugh, M., Kapellusch, J., ... Hegmann, K. (2016). The Association between Cardiovascular Disease Risk Factors and Motor Vehicle Crashes among Professional Truck Drivers. Journal of Occupational and Environmental Medicine / American College of Occupational and Environmental Medicine, 58(8), 828-832. doi:10.1097/ JOM.0000000000000806

Rosso, G. L., Perotto, M., Feola, M., Bruno, G., \& Caramella, M. (2015). Investigating obesity among professional drivers: the high risk professional driver study. Am J Ind Med, 58(2), 212-219. doi:10.1002/ajim.22400

Ruiz, F. J., García-Beltrán, D. M., \& Suárez-Falcón, J. C. (2017). General Health Questionnaire-12 validity in Colombia and factorial equivalence between clinical and nonclinical participants. Psychiatry Res, 256, 53-58. doi:10.1016/j.psychres.2017.06.020

Ruiz, J.I., Arbeláez, V.; Calderón, M. (2006). Escala de Agresividad de Buss y Perry: una comparación de población reclusa y no reclusa. VI Congreso Iberoamericano De Psicología Jurídica. Bogotá 24-26 de noviembre del 2006.

Ruiz, J.I, \& López, C., (2010). Escala de dificultades percibidas para la conducción, hostilidad y extraversión: un análisis correlacional en conductores de Bogotá. Diversitas, 6(2), 449-462.

Ruiz, J.I.; Gómez, I.A.; Beltrán, I.T.; Lamus, D.A., \& Leal-Salazar, L.D. (2014). Representaciones sociales de normas de tránsito, agresividad, facilidad percibida en la conducción, accidentes y multas en conductores de Bogotá, D. C. Revista Criminalidad. 56 (2), 291-307.

Sami, A., Moafian, G., Najafi, A., Aghabeigi, M. R., Yamini, N., Heydari, S. T., \& Lankarani, K. B. (2013). Educational level and age as contributing factors to road traffic accidents. Chin J Traumatol, 16(5), 281-285.

Serge, A. (2015). Relación entre salud y accidentalidad vial en conductores colombianos: estudio desde variables epidemiológicas. Tesis de Maestría en Psicología, Universidad Nacional de Colombia. doi:10.13140/ RG.2.2.30300.18560

Thomas, P., Morris, A., Talbot, R., \& Fagerlind, H. (2013). Identifying the causes of road crashes in Europe. Annals of Advances in Automotive Medicine, 57, $13-22$

Tolon-Becerra, A., Lastra-Bravo, X., \& Flores-Parra, I. (2013). National and regional analysis of road accidents in Spain. Traffic Inj Prev, 14(5), 486-495. doi:10.10 80/15389588.2012.726384

Useche, S. A., \& Ruiz, J.I. (2011). Prevención en seguridad vial: Desarrollo de una estrategia de evaluación para la asignación y renovación de licencias de conducción. Departamento Administrativo de Ciencia, Tecnología e Innovación. Bogotá: Colciencias.

Van Dierendonck, D. (2004) The construct validity of Ryff's Scale of Psychological well-being and its extension with spiritual well-being. Personality and Individual Differences, 36 (3), 629-644.

Vigil-Colet, A., Lorenzo-Seva, U., Codorniu-Raga, M. J., \& Morales, F. (2005). Factor structure of the Buss-Perry aggression questionnaire in different samples and languages. Aggressive Behavior, 31(6), 601-608. doi:10.1002/ab.20097

WHO (1948). Preamble to the Constitution of the World Health Organization as adopted by the International Health Conference; New York. 19-22 June, 1946; signed on 22 July 1946 by the representatives of 61 States (Official Records of the World Health Organization, N², p. 100) and entered into force on 7 April 1948. 